\title{
Ground State and Lowest Eigenvalue of the Laplacian for Non-Compact Hyperbolic Surfaces
}

\author{
Thea Pignataro ${ }^{1, \star}$ and Dennis Sullivan ${ }^{1,2}$ \\ 1 I.H.E.S., F-91440 Bures-sur-Yvette, France \\ ${ }^{2}$ Graduate Center of the City University of New York, New York, NY 10036, USA
}

\begin{abstract}
Let $M$ be a complete Riemannian surface with constant curvature -1 , infinite volume, and a finitely generated fundamental group. Denote by $\lambda(M)$ the lowest eigenvalue of the Laplacian on $M$, and let $\psi_{M}$ be the associated eigenfunction. We estimate the size of $\lambda(M)$ and the shape of $\psi_{M}$ by a finite procedure which has an electrical circuit analogue. Using the Margulis lemma, we decompose $M$ into its thick and thin parts. On the compact thick components, we show that $\psi_{M}$ varies from a constant value by no more than $O(\sqrt{\lambda(M)})$. The estimate for $\lambda(M)$ is calculable in terms of the topology of $M$ and the lengths of short geodesics of $M$. An analogous theorem of the compact case was treated in [SWY].
\end{abstract}

Let $M$ be a complete Riemannian surface with constant curvature -1, infinite volume, and a finitely generated fundamental group. The lowest eigenvalue of the Laplacian, $\lambda(M)$, belongs to $(0,1 / 4]$ and if $\lambda(M)$ belongs to $(0,1 / 4)$, there is a unique positive eigenfunction $\psi_{M}$ for $\lambda(M)$ of $L^{2}$ norm one (see [P1, S, S2]). If one writes $\lambda(M)=D(1-D)$ with $D>1 / 2$ then $D$ is the Hausdorff dimension of the limit set of the Fuchsian group representing $M$. Also the value of $\psi_{M}$ lifted to the unit disk at a point $p$ is just the (packing) Hausdorff $D$-measure of the limit set in the metric on rays from $p$ (see $[\mathrm{S}, \mathrm{S} 2]$ ).

In this paper we will describe a finite procedure for determining the size of $\lambda(M)$ and the shape of $\psi_{M}$. The procedure has an electrical circuit analogue which could, in principle, be used to compute this size and shape.

As a corollary let $\Gamma_{\varepsilon}$ be the Fuchsian group generated by $z \rightarrow-1 / z$ and the translation by $2+\varepsilon$. Our Theorem 1 implies the Hausdorff dimension of the limit set of $\Gamma_{\varepsilon}$ differs from one by the quantity $\sqrt{\varepsilon}$. This estimation implies the derivative of the dimension at the critical value $\varepsilon=0$ is $+\infty$ (see [P2]).

Furthermore, the estimate has the simple interpretation often observed in dynamically defined Cantor sets on the line, namely that when the dimension is

* Present address: Courant Institute, New York University, 251 Mercer Street, New York, NY 10012, USA 


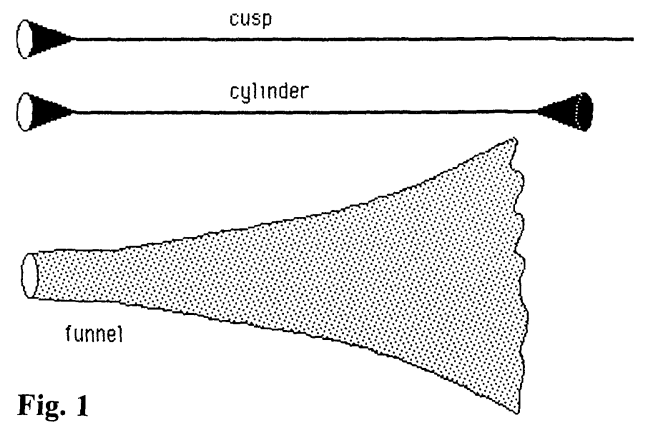

near one, the discrepancy from one is on the order of the size of the largest gap in the Cantor set.

To describe this procedure we divide $M$ into the thick part $M_{T}$ (in which each point is the center of an embedded disk of a definite convenient radius) and the complementary thin part $M_{t}$ (which is proven to consist of cusps, or pseudospheres, and cylinders, which are approximately doubled truncated cusps) see [T].

The thick part $M_{T}$ has finitely many components: some compact of bounded geometry, called nodes here; and others non-compact which, up to bounded pieces, are made of canonical pieces called funnels (see Fig. 2).

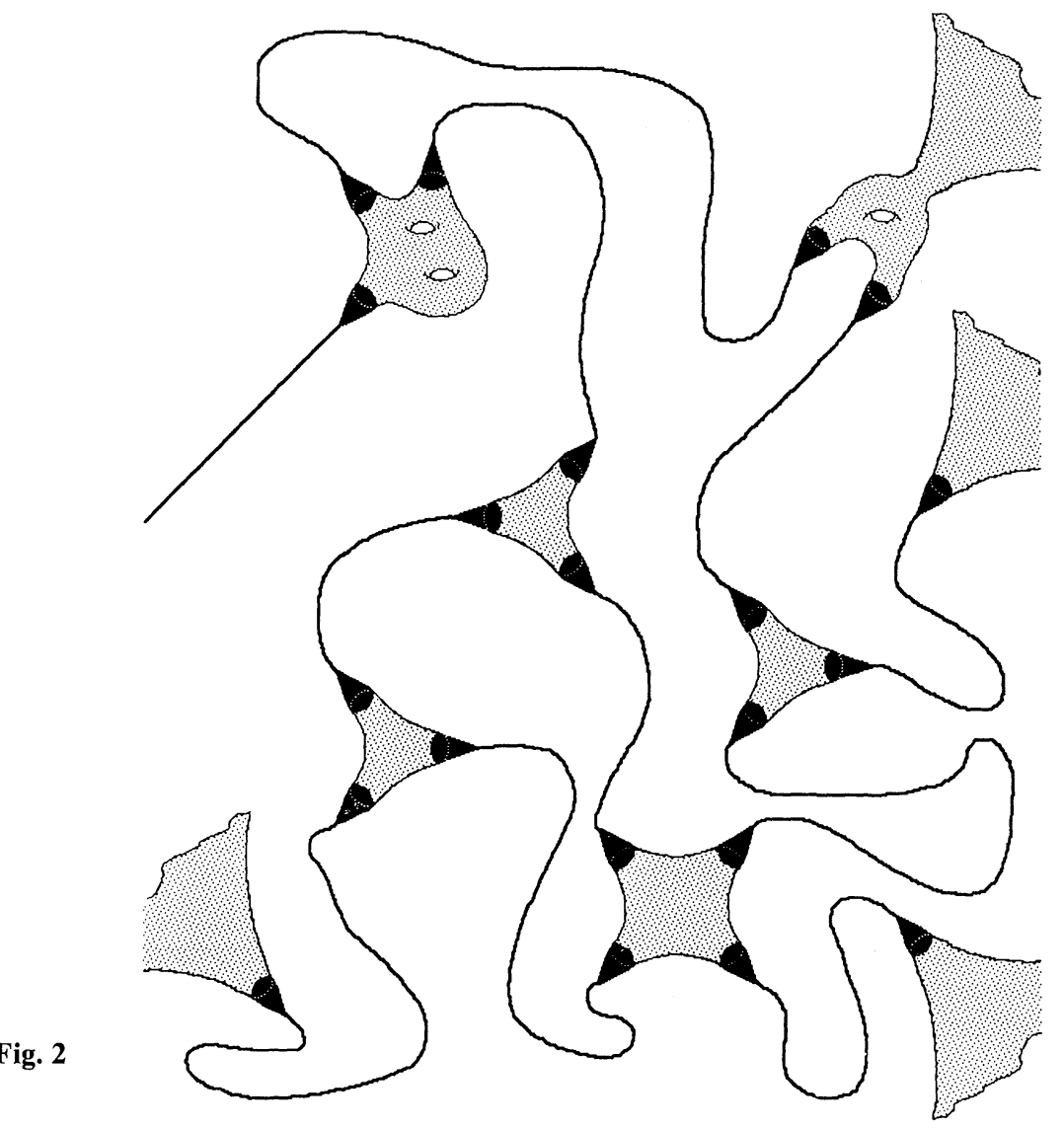




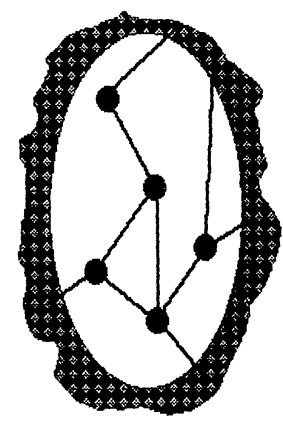

Fig. 3

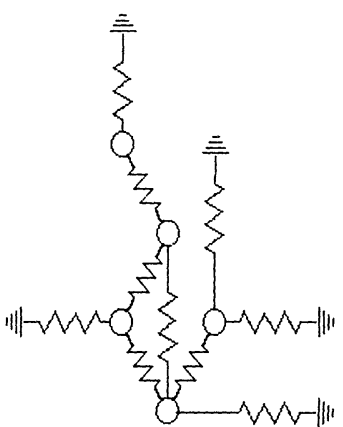

Fig. 4

We associate a finite graph and a circuit diagram to this decomposition as follows. There is one vertex for each compact component of $M_{T}$, and one vertex called ground which is associated to all the non-compact components. There is an edge between two vertices for each cylinder. For reasons which will become clear below, one may forget the cusps in the formation of this graph.

From this graph one forms a circuit diagram where each nodal vertex has a variable voltage with respect to the ground vertex (at zero potential), and each edge becomes a resistor with resistance the reciprocal of the length of the short geodesic of that cylinder.

The finite procedure is to form the quantity

$$
m_{a}=\inf _{f} \sum_{\text {edges }}(\delta f)^{2} \ell
$$

where $f$ is a vertex function of square norm one $\left(\sum_{\text {vertices }} f(V)^{2}=1\right), f$ (ground) $=0$, $\delta f$ (edge $)=$ difference of values of $f$ at either end, and $\ell$ is the length of the edge.

The size of the number $m_{a}$ can be calculated physically as follows: create a voltage of one at a node and using an ammeter record the maximum of (current) ${ }^{2}$ (resistance) across each resistor. The minimum $m_{c}$ over the nodes of these maxima has the same size as $m_{a}$. Denote by $m$ the size of either of these quantities.

Theorem 1. $\lambda(M)$ and $m$ have the same size, i.e. their ratio is bounded above and below by constants depending only on the topology of $M$.

Proof. To see that $m$ is an upper bound we construct a test function. To see that $m$ is a lower bound we use conformal invariance of the energy in dimension two to show that the basic eigenfunction for $\lambda(M)$ resembles this test function.

\section{The Upper Bound}

By definition, $\lambda(M)=\inf _{\psi} \int|\operatorname{grad} \psi|^{2}$, where $\psi$ ranges over smooth functions with compact support and square integral one. Thus upper bounds for $\lambda(M)$ can be found by considering test functions. For example, choose constant functions $V_{1}, V_{2}, \ldots$ for each compact component of $M_{T}$ (the nodes) and choose zero on each non-compact component of $M_{T}$ (the ground). In the unit tubular neighborhood of 
the short geodesic of each cylinder interpolate a linear function between these constants. If $\ell$ is the length of the short geodesic and $V_{1}, V_{2}$ are the constants, the energy $\int|\operatorname{grad} \psi|^{2}$ on this part is about $\left(V_{1}-V_{2}\right)^{2} \ell$. In each cusp we can interpolate the constant to zero so far out in the cusp that the energy is negligible (by the above). If the maximum constant is one, the $L^{2}$ norm of our test function is comparable to one, and the energy is comparable to the maximum of $\left(V_{1}-V_{2}\right)^{2} \ell$ over the cylinders.

If the one occurs at a certain node, the circuit analogue chooses the other voltages $V_{\propto}$ between zero and one to minimize the sum of the energy terms (current $)^{2} \cdot$ resistance which are just the terms $\left(V_{1}-V_{2}\right)^{2} \ell$.

This completes our discussion of the upper bound.

\section{The Lower Bound}

Now the fun begins. We will show that the actual basic eigenfunction $\psi_{M}$ resembles one of the test functions above, thus proving that $m$ gives a lower bound for $\lambda(M)$.

We suppose $\lambda(M)$ is much less than $m$, for otherwise we would be done.

1) Since $\int\left(\operatorname{grad} \psi_{M}\right)^{2}=\lambda(M) \ll m$, the $L^{2}$ norm of $\left(\operatorname{grad} \psi_{M}\right) \ll \sqrt{m}$. Thus the derivative of $\operatorname{grad} \psi_{M} \ll \sqrt{m}$ at any point of $M_{T}$ by Harnack. Thus if $\operatorname{grad} \psi_{M}$ is not much smaller than $\sqrt{m}$ at a point of $M_{T}$ then $\operatorname{grad} \psi_{M}$ would have the size $\sqrt{m}$ on a ball of definite radius. (See Harnack principle in Appendix.) So the $\int\left(\operatorname{grad} \psi_{M}\right)^{2}$ would have at least the size $m$, contradicting our assumption $\lambda(M) \ll m$. Thus we deduce, using the uniform bounds on diameters of compact components of $M_{T}$,

2) the variation of $\psi_{M}$ on each compact component of $M_{T}$ is $\ll \sqrt{m}$.

3) Define a test function $f$, as above, where $V_{i}$ is the average of $\psi_{M}$ on the corresponding component of $M_{T}$. We claim a) the $L^{2}$ norm of $f$ is not much smaller than the $L^{2}$ norm of $\psi_{M}$ which is one, b) the energy of $f$ is not much bigger than the energy of $\psi_{M}$ which is $\lambda(M)$. From the claim we deduce the lower bound.

4) Proof of b). Consider any cylinder. Let $\ell$ be its waist size and suppose $f$ is $V_{1}$ at one end and $V_{2}$ at the other. Recall the energy of $f$ in the cylinder is $\left(V_{1}-V_{2}\right)^{2} \ell$. Let $C$ be the cylinder where this energy contribution to $f$ is a maximum. Then of course, the total energy of $f$ is comparable to $\left(V_{1}-V_{2}\right)^{2} \ell$, since there is a fixed number of cylinders.

Now $\psi_{M}$ must also vary across the cylinder by 2). Namely if $V_{1}<V_{2}, \psi_{M} \leqq C_{1}$ $=V_{1}+($ small fraction of $\sqrt{m})$ at the $V_{1}$ end and $\psi_{M} \geqq C_{2}=V_{2}-($ small fraction of $\sqrt{m}$ ) at the $V_{2}$ end, using 2). By Lemma 2, the energy of $\psi_{M}$ in the cylinder is at least $\left(C_{1}-C_{2}\right)^{2} \ell$. (An easy calculation shows the modulus of a cylinder of waist $\ell$ is $1 / \ell$.) Since $\left(V_{1}-V_{2}\right)^{2} \ell$ is comparable to $m$, so is $\left(C_{1}-C_{2}\right)^{2} \ell$. This proves b).

The proof of a) takes several steps. We continue to pretend that $\lambda(M) \ll m$. This assumption now entails that each $V_{i}$ is much less than one, for otherwise $f$ determines a normalized test function showing $\lambda(M)$ and $m$ are comparable.

We will use this information to show each contribution to the $L^{2}$ norm of $\psi_{M}$ is small, contradicting the fact that the $L^{2}$ norm of $\psi_{M}$ is one.

5) (the structure of $\psi_{M}$ in a cusp) If $\psi_{M}(x)$ is $c$ for $x$ in the boundary of a cusp, then $\psi_{M}$ has the size of $c$ on the entire boundary (Harnack). By [P1] and [S] there is an upper bound $\psi_{M}(y) \leqq c \exp (1-D) R$, where $R=\operatorname{distance}(x, y)$ and $\lambda(M)$ is 
$D(1-D), D>1 / 2$. The width of the cusp at distance $R$ is $e^{-R}$, thus the square integral of $\psi_{M}$ in the cusp has the size $c^{2}$. If the cusp touches a compact component of $M_{T}$, then $c$ is comparable to one of the $V_{i}^{\prime}$ s and thus the $L^{2}$ norm of $\psi_{M}$ in the cusp is small. If the cusp touches a non-compact component we deduce this contribution to the $L^{2}$ norm of $\psi_{M}$ is small using 6) below.

We note that the estimate is easy to derive using the fact that $\psi_{M}$ belongs to $L^{2}$, and an argument like 7) below. However, this estimate is proved independently of this fact in [P1] or [S] to show $\psi_{M}$ belongs to $L^{2}$.

6) (structure of $\psi_{M}$ in a non-compact component of $M_{T}$ ) We study $\psi_{M}$ on the non-compact part of $M_{T}$ by isolating each end by closed geodesics. There are two cases: i) the component of $M_{T}$ containing that end is itself an annulus entirely contained in a larger annulus between a (short) closed geodesic and infinity, ii) the end of $M_{T}$ may be isolated by a geodesic in that component of $M_{T}$ (see Fig. 5).

In either case if $k$ is the value of $\psi_{M}$ at a point $x$ of that geodesic, then for any $y$ between the geodesic and infinity, $\psi_{M}(y)$ has the size $k(\exp -D R)$ where $R=\operatorname{distance}(x, y)$ and $D=1 / 2+\sqrt{[1 / 4-\lambda(M)]}$ (see [S]). Thus in both cases i) and ii) we see that the contribution to the $L^{2}$ norm of $\psi_{M}$ from a funnel is comparable to the size of $\psi_{M}$ in the boundary of the funnel. (Recall that the boundary component has diameter of size one.) If $c$ is this value, by Lemma 1 the energy of $\psi_{M}$ in the funnel is at least size $c^{2}$. Thus the size of $c$ is smaller than $\sqrt{\lambda}$, which is smaller than one.

We note that this estimate follows easily from the geometric observation that as a point moves away from the closed geodesic its image in the universal cover is moving away from the convex hull of the limit set (where the boundary measure of $\psi_{M}$ is concentrated) at the same rate, up to an additive constant.

7) (structure of $\psi_{M}$ in a cylinder) Average $\psi_{M}$ over the rotations of the cylinder to construct a new function $\psi$. By Harnack $\psi$ has the same size as $\psi_{M}$. Also, $\psi$ satisfies a second order differential equation with two independent solutions $\psi_{+}$ and $\psi_{-}$. When one symmetric half of the cylinder is close to a cusp these solutions are close to $y^{D}$ and $y^{1-D}$ (upper half plane model). If $c<1$ is the value of $\psi$ on the boundary and the $L^{2}$ norm of $\psi$ is less than or equal to one, a calculation (see below) using $\psi=A_{+} \psi_{+}+A_{-} \psi_{-} \sim A_{+} y^{D}+A_{-} y^{1-D}$ yields boundary values of $\psi_{M}$ and the eigenvalue controls its $L^{2}$ norm on a cylinder.

Here is the calculation: If in the upper half plane model $f=A_{+} y^{D}+A_{-} y^{1-D}$ on $0 \leqq x \leqq 1,1 \leqq y \leqq 1 / \ell$ (recall this rectangle is approximately isometric to half of a

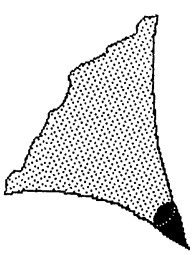

Fig. 5

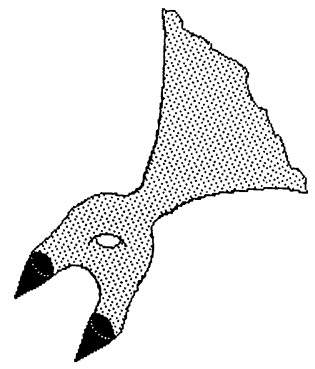


cylinder with short geodesic of length $\ell$ ) then, letting $L=1 / \ell$, the $L^{2}$ norm of $f$ is

$$
\int f^{2} y^{-2} d x d y=\int_{1}^{L}\left(A_{+}^{2} y^{2 D-2}+2 A_{+} A_{-} y^{-1}+A_{-}^{2} y^{-2 D}\right) d y,
$$

or $(*)(\sim 1) A_{+}^{2} L^{2 D-1}+2 A_{-} A_{+} \log L+A_{-}^{2}(\sim 1)$. We now calculate the energy in the half cylinder. In the upper half plane model, $\operatorname{grad}\left(y^{\alpha}\right)=y \cdot\left(\alpha y^{\alpha-1}\right)=\alpha y^{\alpha}$. Thus

$$
\begin{aligned}
\text { energy } & =\int_{1}^{L}\left[\operatorname{grad}\left(A_{+} y^{D}+A_{-} y^{1-D}\right)\right]^{2} y^{-2} d y \\
& =\int_{1}^{L}\left[D A_{+} y^{D}+(1-D) A_{-} y^{1-D}\right]^{2} y^{-2} d y \\
& =\int_{1}^{L}\left[D^{2} A_{+}^{2} y^{2 D-2}+D(1-D) A_{+} A_{-} y^{-1}+(1-D)^{2} A_{-}^{2} y^{-2 D}\right] d y \\
& =(\sim 1) A_{+}^{2} L^{2 D-1}+\lambda(M) A_{+} A_{-} \log L+(1-D)^{2} A_{-}^{2}(\sim 1) .
\end{aligned}
$$

Since $\psi$ is positive and $A_{+}+A_{-}=c$, there are three possibilities: 1$) A_{+}$and the magnitude of $A_{-}$have the same size, 2) $A_{+} \sim c$ and is much greater than the magnitude of $A_{-}$, or 3 ) the magnitude of $A_{+}$is much smaller than that of $A_{-}$, which is approximately $c$. In cases 1) and 2) using the facts that $D>\frac{1}{2}$ and $L$ is large, we see that the dominant term is $A_{+}^{2} L^{2 D-1}$ in both the energy and the $L^{2}$ norm expressions. Thus the $L^{2}$ norm and the energy in the half cylinder have the same size and this size is dominated by $\lambda(M)$ which is small. In case 3$)$ using the above facts and assuming that $A_{+} A_{-} \log L$ dominates the $L^{2}$ norm expression leads to the contradiction that $A_{-} \gg c$. Thus either $\lambda(M)$ controls the $L^{2}$ norm, as in the previous cases; or, if $A_{+}$is small enough, $A_{-}^{2}$ and hence $c$ controls it. This completes the proof of Theorem 1 .

Shape of the Basic Eigenfunction $\psi_{M}$. We summarize the information about $\psi_{M}$ derived from the proof of Theorem 1.

1) On each compact component of $M_{T}, \psi_{M}$ varies from a constant value by no more than $O(\sqrt{\lambda})$. A definite proportion of the $L^{2}$ norm of $\psi_{M}$ lives on these compact components.

2) In the cusps, cylinders and funnels, the size of $\psi_{M}$ is determined by the sizes on the boundary and the eigenvalue.

3) The test function produced from the constants on the compact components of the thick part by linear interpolation on the cylinders has the correct size energy and $L^{2}$ norm to compute the magnitude of $\lambda(M)$.

4) We have not shown that the energy of $\psi_{M}$ is situated in the same place as the energy of the test function.

\section{Appendix}

Harnack Principle. 1) The value of a positive eigenfunction at a point controls its values on a unit neighborhood. 2) A bound on a disk neighborhood for any eigenfunction controls any finite number of derivatives on a centered disk of half the radius. 3) The $L^{2}$ norm of the gradient of an eigenfunction on a disk of definite 
size controls a finite number of derivatives on an interior centered disk of half the radius.

The basis for these general facts can be found in references on elliptic equations.

Lemma 1. Let $M$ be a Riemannian surface homeomorphic to $S^{1} x[0, \infty)$. Let $\psi$ be a positive smooth function greater than or equal to a constant $C$ on $\partial M$ and such that $\psi \rightarrow 0$ as $x \rightarrow \infty$. Then energy $(\psi) \geqq C^{2} / \operatorname{modulus}(M)$.

Proof. We assume that modulus $(M)<\infty$ and note that the energy of a function is a conformal invariant of the metric. Let $h$ be the harmonic function on $M$ with the same boundary values as $\psi$ and which vanishes at infinity. Recall that energy $(h)$ $\leqq \operatorname{energy}(\psi)$.

By Sard's theorem, there exists $C_{0}$ arbitrarily close to $C, C_{0}<C$, for which the level set $\xi=\left\{x \in M: h(x)=C_{0}\right\}$ is a compact one-manifold. If this one-manifold separates an interior piece of $M$, then $h$ is identically constant. Thus $\xi$ is a connected curve separating $\partial M$ from infinity. Let $M_{0}$ be the subannulus between $\xi$ and infinity. Note that modulus $\left(M_{0}\right) \leqq \operatorname{modulus}(M)$ and $h$ is constant on $\partial M_{0}$.

We can map $M_{0}$ conformally to a right circular cylinder. On the cylinder, the function $h$ becomes the linear function with energy $C^{2}$ (modulus of the cylinder) ${ }^{-1}$.

By a similar type of reasoning, we obtain

Lemma 2. If $M$ is a Riemannian surface homeomorphic to $S^{1} x[0,1]$ and $\psi$ is a positive smooth function greater than or equal to a constant $C_{1}$ on one boundary component of $M$ and less than or equal to another constant $C_{2}<C_{1}$ on the other boundary component, then

$$
\text { energy }(\psi) \geqq\left(C_{1}-C_{2}\right)^{2} / \operatorname{modulus}(M) .
$$

Acknowledgement. a) We are indebted to Lori Sullivan for 1) the systems work to produce this paper on the Apple Macintosh, 2) the artwork in the figures.

b) The paper [SWY] came to our attention at the end of the writing. They give bounds in a form equivalent to ours for the first $2 g-3$ eigenvalues of a compact surface of genus $g$ and negative curvature.

\section{References}

[P1] Patterson, S.J.: The limit set of a Fuchsian group. Acta Math. 136, 241-273 (1976)

[P2] Pignataro, T.: Hausdorff dimension, spectral theory and applications to the quantization of geodesic flows on surfaces of constant negative curvature. Thesis, Princeton University (1984), Sects. 3.2 and 4.4

[S] Sullivan, D.: Entropy, Hausdorff measures old and new and the limit sets of geometrically finite Kleinian groups. Acta Math. 153, 259 (1984)

[S2] Sullivan, D.: Related aspects of positivity, ground states, Hausdorff dimension, complementary series, ..., preprint IHES 1983

[T] Thurston, W.: Topology and geometry of three-manifolds. Princeton University 1979

[SWY] Schoen, R., Wolpert, S., Yau, S.-T.: Geometric bounds on the low eigenvalues of a compact surface. AMS Proc. Symp. Pure Math. Vol. 36 (1980)

Communicated by A. Jaffe

Received October 1, 1985 
